\title{
Mobile Near Infrared Spectrometer with a MEMS-FPI Sensor
}

\author{
Artem Ivanov ${ }^{1}$, Arne Kulinna ${ }^{1}$ \\ ${ }^{1}$ Landshut University of Applied Sciences, Am Lurzenhof 1, 84036 Landshut, Germany \\ Artem.Ivanov@haw-landshut.de
}

\begin{abstract}
Summary:
A fast, user friendly and inexpensive hand-held NIR Spectrometer is described. Spectra in the range of $1550 \mathrm{~nm}-1850 \mathrm{~nm}$ are acquired using a MEMS-FPI spectrum sensor. Device control and visualisation of data is accomplished using a software application running on Windows- or Android-devices. Implementation details and experimental results are discussed.
\end{abstract}

Keywords: NIR, Spectrometer, hand-held, MEMS-FPI, screening.

\section{Introduction}

Currently just a few mobile NIR spectrometers are available on the market. These devices focus on use in quality control for agriculture and food industry as well as in the recycling sector [1, 2]. Presented spectrometer was built during a bachelor thesis project with the main goal to develop a low cost hand-held device for measuring reflection Spectra in NIR region in combination with a smartphone or a PC. As the spectrum sensor a Hamamatsu MEMS-FPI (Fabri-Perot Interferometer) was chosen [3]; the manufacturer proposes it for applications like screening of plastics and textiles, moisture detection and ingredient analysis of food. Starting from the $3^{\text {rd }}$ quarter of 2020 Hamamatsu also offers complete spectroscopic modules with MEMS-FPI spectrum sensors [4].

The presented mobile device features a C13272-03 spectrum sensor working in the wavelength range from $1550 \mathrm{~nm}$ to $1850 \mathrm{~nm}$ [5], a control circuitry, a secondary Li-ion cell, USB connection for data communication and battery charging and wireless connectivity via Bluetooth. The device is controlled from the cross-platform software app which offers an easy access to all necessary functions for taking, saving and loading spectra. It runs on Windows- or Androidbased systems (Fig. 1).

\section{Description of the System}

The tuneable MEMS-FPI filter is controlled by the applied voltage in the range from $23 \mathrm{~V}$ to $38 \mathrm{~V}$; its spectral resolution lies below $15 \mathrm{~nm}$ FWHM. To acquire a spectrum the current of the integrated photodiode is recorded for different values of control voltage. In the presented device all necessary tasks are performed by microcontroller-based electronics. Thanks to a high number of available peripherals of the used controller
(PIC24FJ128GC006) it was possible to implement the majority of functions in a single chip.

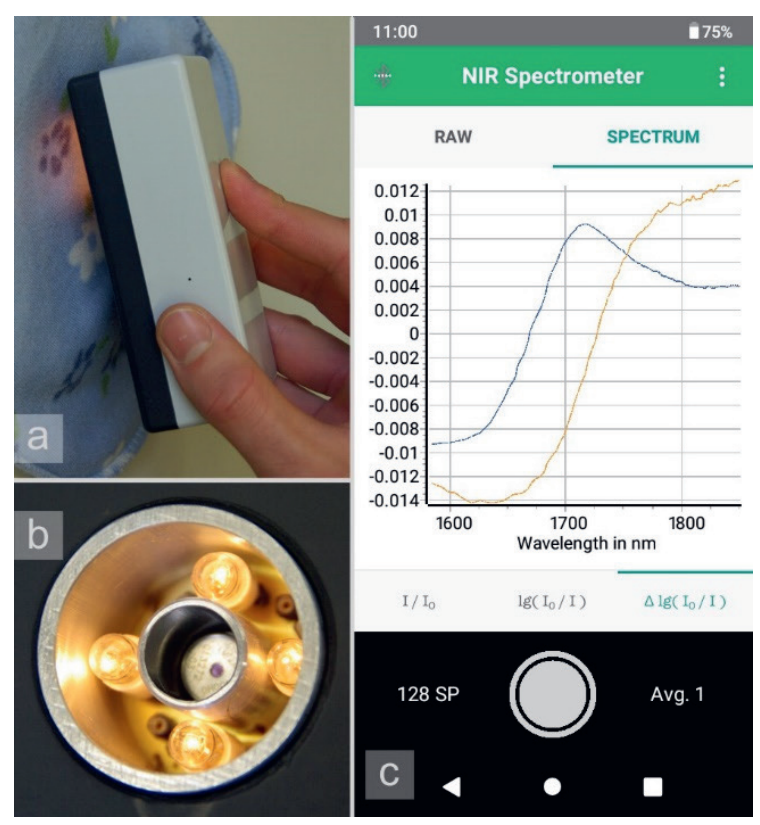

Fig. 1. The mobile NIR spectrometer (a), a close-up view of the illumination optics (b) and a screenshot of the visualization and control app (c) showing spectra of PET (blue) and HDPE (red).

The control voltage for the MEMS-FPI filter is generated internally using a step-up converter and is ramped during a spectral scan; the number of steps is set in the app. During every step $1 \mathrm{~ms}$ time is allowed for the mirror of the MEMS-FPI filter to stabilise, afterwards the photodiode signal is acquired by a 16-bit sigma-delta ADC for $1 \mathrm{~ms}$. The total time needed for one spectrum scan depends on the required number of points in the spectrum, for example one scan with 128 points takes $0.25 \mathrm{~s}$. Currently the number of samples can be set in the range between 64 and 1024 which stands for the equivalent step 
of $5 \mathrm{~nm}$ to $0.3 \mathrm{~nm}$ wavelength. To reduce the noise several scans can be performed and averaged as set in the app.

Standard miniature T-13/4 bi-pin base incandescent lamps are used as the light source, the device can be equipped with up to eight lamps. Based on their intensity to current ratio the lamps of 7361 type ( $5 \mathrm{~V} / 60 \mathrm{~mA}$ nominal, driven at $3.15 \mathrm{~V} / 45 \mathrm{~mA}$ ) were chosen. In case of using only one lamp, a lamp of type 7868 (2.5 V/0.35 A nominal) driven at $2.5 \mathrm{~V}$ or $2.0 \mathrm{~V}$ is suggested for a higher intensity.

The system uses simple reflective optics for illumination of the test sample in front of the sensor. It consists of two blank aluminium tubes and the metallisation of the circuit board below the lamps and is used to direct the light onto the sample while obstructing the direct light path between the lamps and the detector (Fig. 1 b). An additional aperture in front of the sensor can be used to restrict the incidence angle. This lowers the signal amplitude but improves the spectral resolution. A significant positive effect on the obtained spectra of plastics due to the installed aperture was observed, while spectra of textiles remained almost unchanged. Such beam aperture can be added to the design in form of a cap either mounted into the interior tube or placed directly on the sensor housing.

According to [4] it is important to track the temperature of the spectrum sensor to compensate for the temperature induced shifts in the transmission wavelength of the MEMS-FPI filter and to avoid the pull-in of the upper interferometer mirror which can destroy the sensor. This function is accomplished in the presented system by a continuous measurement of integrated thermistor resistance.

The acquired spectrum data can be visualised in the control app in four different representation variants: as raw data in ADC counts, as transmittance $\left(T=/ / I_{0}\right)$ and absorbance $(A=\lg (1 / T))$ as well as the relative absorbance (with constant offsets eliminated). Currently no additional evaluation of spectra is performed. The measurement of the reference spectrum $\left(I_{0}\right)$ is carried out with a "white" reflector (e.g. alumina or a matted metal sample) and contains a dark signal measurement taken with the light source switched off.

\section{Results and Discussion}

Notwithstanding the relative simplicity of the presented device SNR of about $10^{4}$ was achieved; spectra of different materials can be acquired in a fast and simple manner. Fig. 2 gives exemplary results for three textile materials showing clear differences in the measured spectral range.

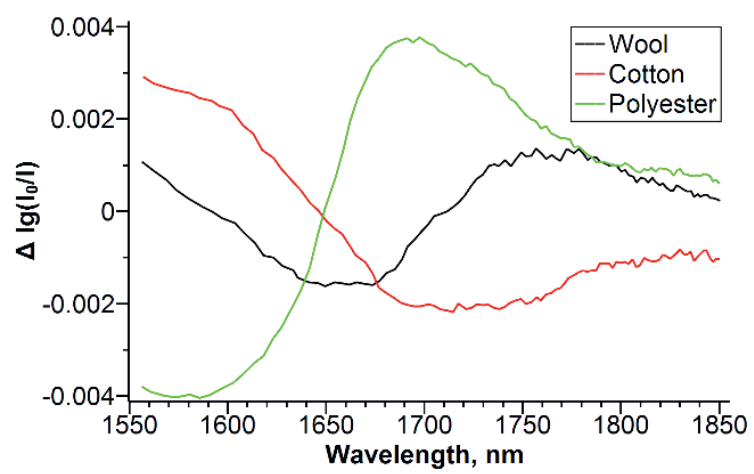

Fig. 2. Spectra of three textile materials measured with 128 sample points in ten scans (spectrum acquisition time $2.5 \mathrm{~s}$ ).

In a plastic sorting application the thickness of the typical items (soft-drink bottles, yoghurt cups, etc.) is low, generally leading to a low reflection and poor spectral data in a direct measurement. It has proven useful to conduct such measurements on a reasonably white surface taking its reflection spectrum as the reference. Spectra acquired in this setup are not strictly reflective but represent a mix of transmission and reflection measurements. Nonetheless, the spectral features remained similar to the pure reflection of thick samples for all tested materials. In tests with textile samples the reflection of the sample alone was always sufficiently high.

The presented project demonstrates a possibility to build a low cost mobile NIR spectrometer (the total hardware effort well below $100 €$ in addition to the spectrum sensor) capable of fast distinguishing between different sorts of materials. In the next steps optimisations of the illumination optics and control circuitry as well as integration of spectral evaluation features and material identification algorithms in the software app are planned.

\section{References}

[1] Consumerphysics.com, SClO by Consumer Physics. [Online]. Available: https://www.consumerphysics.com. [Accessed: 27-Nov-2020]

[2] TrinamiX, Mobile NIR Spectroscopy Solutions. [Online]. Available: https://trinamixsensing.com/nir-spectroscopy/. [Accessed: 27-Nov2020]

[3] Hamamatsu, MEMS-FPI spectrum sensors. [Online]. Available: https://www.hamamatsu.com/eu/en/product/optical-sensors/spectrometers/mems-fpi-spectrum-sensor/index.html. [Accessed: 27-Nov-2020]

[4] Hamamatsu, MEMS-FPI spectroscopic module. [Online]. Available: https://www.hamamatsu.com/eu/en/product/optical-sensors/spectrometers/spectroscopic-modules/index.html. [Accessed: 27-Nov-2020]

[5] Hamamatsu, MEMS-FPI Spectrum Sensor C13272-03, Datasheet (Feb. 2020) 